\title{
Incidence of Accessory Brachialis Muscle, Variations in its Insertion and Relation with Surrounding Neurovascular Structures
}

\author{
M. Tonse, Mangala M. Pai, Latha V. Prabhu, B. V. Murlimanju, Lakshmisha Y. Rao \\ Department of Anatomy, Kasturba Medical College, Mangalore, Manipal Academy of Higher Education, Manipal, \\ Karnataka, India
}

\author{
CORRESPONDING AUTHOR: \\ Mangala M. Pai \\ Department of Anatomy \\ Kasturba Medical College \\ Center for Basic Sciences \\ Bejai, Mangalore 575004 \\ Karnataka, India \\ E-mail: mangala.pai@manipal.edu \\ DOI: \\ 10.32098/mltj.04.2021.21
}

LEVEL OF EVIDENCE: 4

\begin{abstract}
SUMMARY
Background. Accessory brachialis ( $\mathrm{AcBr}$ ) muscle can compromise the surrounding neurovascular structures due its variable insertion. In this context, the objective of the present study was to determine the incidence of $\mathrm{AcBr}$ muscle, variation of its insertion and relation with surrounding neurovascular structures.

Methods. The study was performed in 84 formalin fixed human cadaveric upper limb specimens, which were available in the department of anatomy. The insertion pattern of the AcBr muscle was divided into 5 types (type 1, type 2, type $3 \mathrm{a}$, type $3 \mathrm{~b}$ and type 4 ).

Results. It was observed that, AcBr muscle was present in $46(54.8 \%)$ cases. In 31 cases $(67.4 \%)$, this muscle inserted into the main tendon of brachialis muscle (type 1$)$. It joined the tendon of biceps brachii (type 2 ) in 6 cases (13\%). In 7 cases $(15.2 \%)$, it gave a slip which joined the supinator muscle after passing deep to the radial recurrent vessels (type $3 a)$. In a single case $(2.2 \%)$, the slip merged with the supinator after passing superficial to the radial recurrent vessels (type $3 \mathrm{~b}$ ). In one case $(2.2 \%)$, it gave muscular fibres which crossed the radial nerve and merged with the brachioradialis muscle (type 4).

Conclusions. The present study observed higher frequency of incidence of AcBr muscle, however, the limitation of this study is that the small number of specimens studied. The findings will be more accurate with a larger sample size. The gender-based comparison was not performed, since the study involved disarticulated upper extremities.
\end{abstract}

\section{KEY WORDS}

Anatomic variation; biceps brachialis; muscles; nerve compression syndromes; orthopedic disorders.

\section{BACKGROUND}

Accessory muscles may produce a palpable swelling or could lead to pressure effect on the adjacent neurovascular structures. Accessory muscles at the arm region may contribute to neurovasculopathy by compressing the brachial artery and median nerve $(1,2)$. In clinical cases, where an apparent cause for such symptoms is unknown, evaluation of accessory muscles might help in the accurate diagnosis. In the radiological setup, study of cross-sectional images can reveal the accessory muscles and help in differentiating them from the soft-tissue tumors (3). Entrapment of the vessels and nerves can happen, because these accessory muscles pass anterior to them. The accessory muscles of the arm region have clinical implications and to be considered as the etiolo- gy in median, ulnar and medial cutaneous nerve of forearm paralysis on few occasions. They can also cause symptoms of compression of brachial vessels (4). In the surgical planning, if there is an anatomical variation, it becomes hard to identify the topographical location of the nerves in the muscular compartments, which complicates the surgical approach (5). It was reported that the variations of brachialis muscle are rare in the scientific literature (6). Accessory brachialis $(\mathrm{AcBr})$ muscle, if present can compress the surrounding neurovascular structures in the supracondylar region (6-8). It can also affect the movements of the elbow joint like flexion, extension, pronation and supination. The $\mathrm{AcBr}$ muscles are grouped together as the brachialis anticus (9). The knowledge of unusual insertion of brachialis muscle is 
important during the surgical approach to the elbow joint (10). It was reported that the distal tendon of $\mathrm{AcBr}$ may split and enclose the median nerve. This could lead to the symptoms of median nerve entrapment (7). Careful examination of the cubital tunnel for an accessory muscle might help, which can be a causative factor in the musculoskeletal problem of the upper limb (3). Because of these clinical relevance, the present anatomical investigation was conducted to determine the incidence of $\mathrm{AcBr}$ muscle, variability in its insertion pattern and to study the relation to the surrounding neurovascular structures.

\section{MATERIALS AND METHODS}

This research work was executed in 84 formalin fixed human cadaveric upper extremities. Among them, 48 were right upper limbs and 36 belonged to left upper limbs. The gender of the cadavers was not taken into consideration. The extremities were from the donated cadavers, which were available in the department of anatomy. The present study was assessed and certified as approved (IEC KMC MLR 08-18/190) by the ethics committee of our medical college. We state that the present study is as per the guidelines of international ethical standards suggested by Padulo et al. (11). The cubital fossa and arm were meticulously dissect- ed and the floor of cubital fossa was reached to expose the brachialis and supinator muscles. The presence of $\mathrm{AcBr}$ was checked next to the brachialis in the arm and cubital fossa. If $\mathrm{AcBr}$ is present, its distal attachment was dissected and noted. The photographs were taken after cleaning the structures. The relation of $\mathrm{AcBr}$ with the surrounding neurovascular structures was also studied.

The present study classified the AcBr into 5 types, depending on its insertion pattern as below (figure 1):

- type 1: AcBr inserting into the tendon of brachialis muscle;

- type 2: AcBr inserting into the tendon of biceps brachii;

- type 3a: AcBr inserting into the supinator muscle after passing deep to the radial recurrent vessels;

- type 3b: AcBr inserting into the supinator after passing superficial to the radial recurrent vessels;

- type 4: slip of $\mathrm{AcBr}$ crossing the radial nerve and inserting into the brachioradialis muscle.

\section{RESULTS}

According to observations of the present study, $\mathrm{AcBr}$ muscle was present in $46(54.8 \%)$ cases. It was observed in 27 right sided and 19 left sided upper extremities. The present study revealed that the $\mathrm{AcBr}$ was more prevalent in

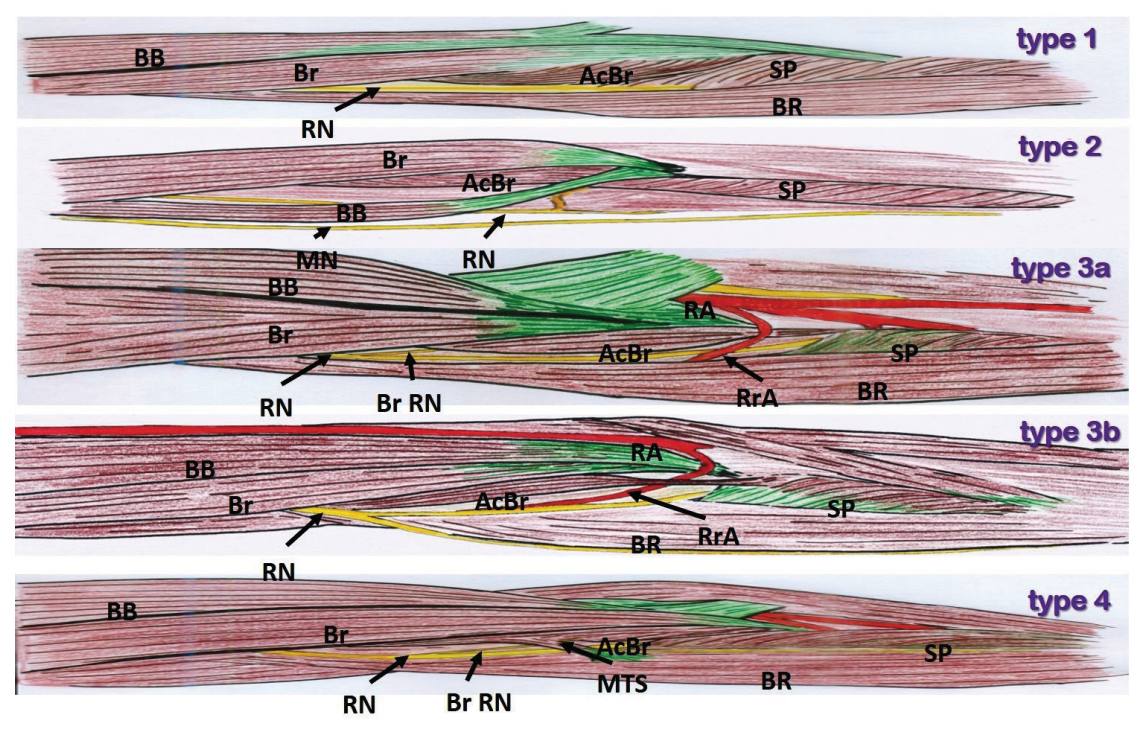

Figure 1. Various types of $\mathrm{AcBr}$ muscle observed in the present study, inserting into brachialis (type 1); biceps brachii (type 2); supinator and passing deep to radial recurrent vessels (type 3a); supinator and passing superficial to radial recurrent vessels (type 3b); brachioradialis (type 4).

AcBr: accessory brachialis; BB: biceps brachii; Br: brachialis; SP: supinator; BR: brachioradialis; RN: radial nerve; MN: median nerve; Br RN: branch of radial nerve; RA: radial artery; RrA: radial recurrent artery; MTS: musculo-tendinous slip. 
the right upper extremity in comparison to the left side (28:18). In 31 cases $(67.4 \%)$, the $\mathrm{AcBr}$ was inserting into the brachialis muscle (type 1, figure 2). Among them 16 were right sided and 15 were left sided upper extremities. AcBr joined the tendon of biceps brachii in 6 cases $(13 \%)$, which included 4 right and 2 left sided upper limbs (type 2, figure 3). In 7 (15.2\%) specimens ( 6 right sided and 1 left sided), AcBr gave a slip, which joined the supinator muscle after passing deep to the radial recurrent vessels (type 3 a, figure 4). In only one case (right sided), the slip merged with the supinator $(2.2 \%)$ after passing superficial to the radial recurrent vessels (type $3 \mathrm{~b}$, figure 5 ). In one right upper limb $(2.2 \%)$, musculotendinous fibres of $\mathrm{AcBr}$ crossed the radial nerve and merged with the brachioradialis muscle (type 4 , figure 6). The frequency distribution of variability in the insertion of $\mathrm{AcBr}$ of this study is represented in figure 7 . It was observed that, all the cases of $\mathrm{AcBr}$ were innervated by the branch of radial nerve, which was originating at the distal part of the arm.

\section{DISCUSSION}

The muscular variations can be understood by studying the embryological development of the muscles. The upper limb musculature develops from the limb bud at the level of lower six cervical and upper two thoracic segments. In the fifth week of gestation, the development of forelimb muscles take place in the mesenchyme of the para-axial mesoderm. The somites will be partitioned into the sclerotome, myotome and dermatome. Muscle primordia develop from the posterolateral side of the somite cells, which migrate into the limb buds around $28^{\text {th }}$ day of intrauterine life (12). The myotomes will develop as the muscles as numerous growth factors are secreted by the cells in the proximal limb

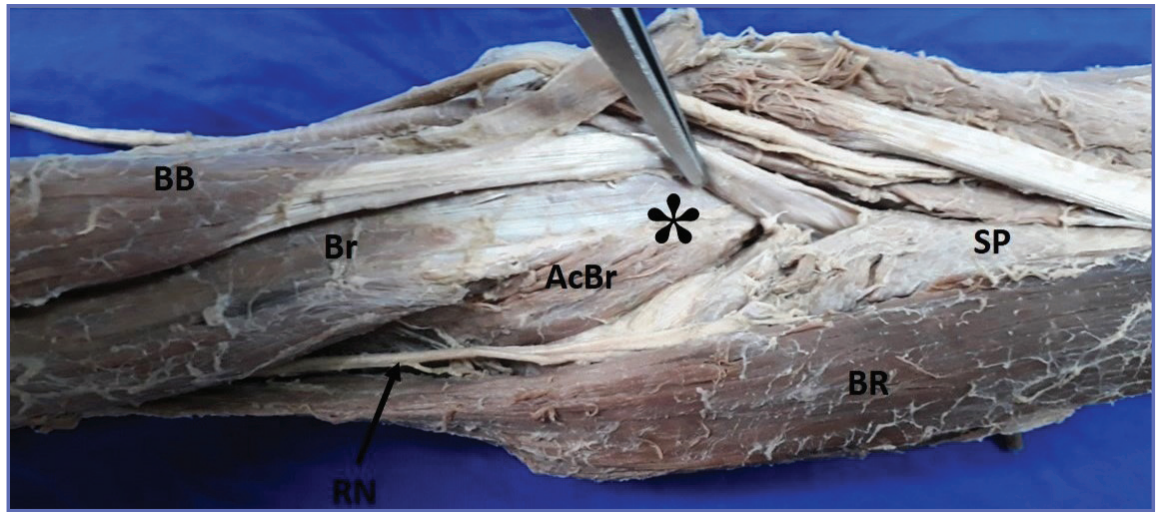

Figure 2. AcBr inserting into the tendon of $\mathrm{Br}$ (67.4\%; type 1).

BB: biceps brachii; Br: brachialis; AcBr: accessory brachialis; BR: brachioradialis; SP: supinator; $\mathrm{RN}$-radial nerve. *Insertion of $\mathrm{AcBr}$ into $\mathrm{Br}$.

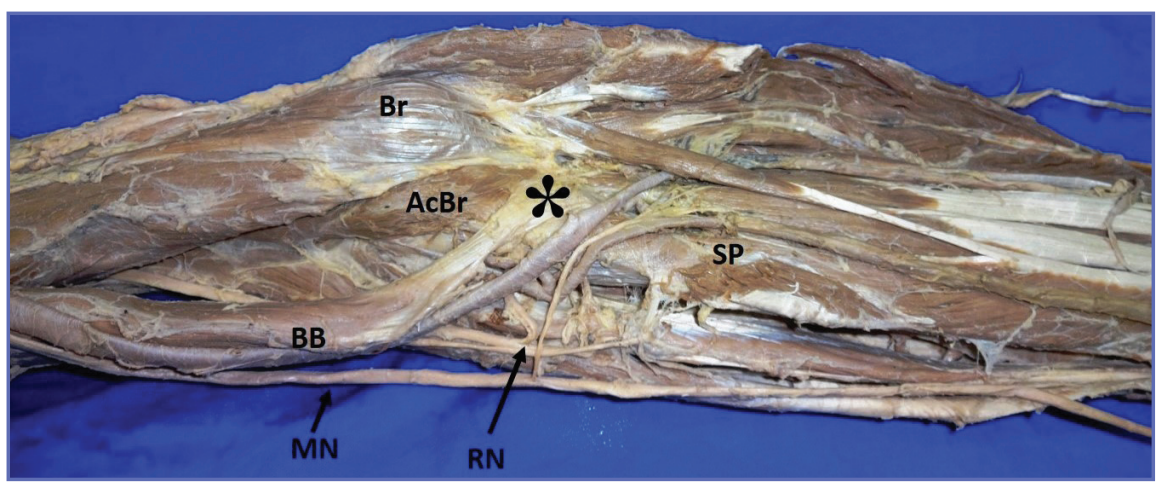

Figure 3. AcBr inserting into the tendon of $B B$ (13\%; type 2).

$\mathrm{BB}$ : biceps brachii; Br: brachialis; AcBr: accessory brachialis; SP: supinator; RN: radial nerve; $\mathrm{MN}$ : median nerve. *Insertion of $\mathrm{AcBr}$ into $\mathrm{BB}$.

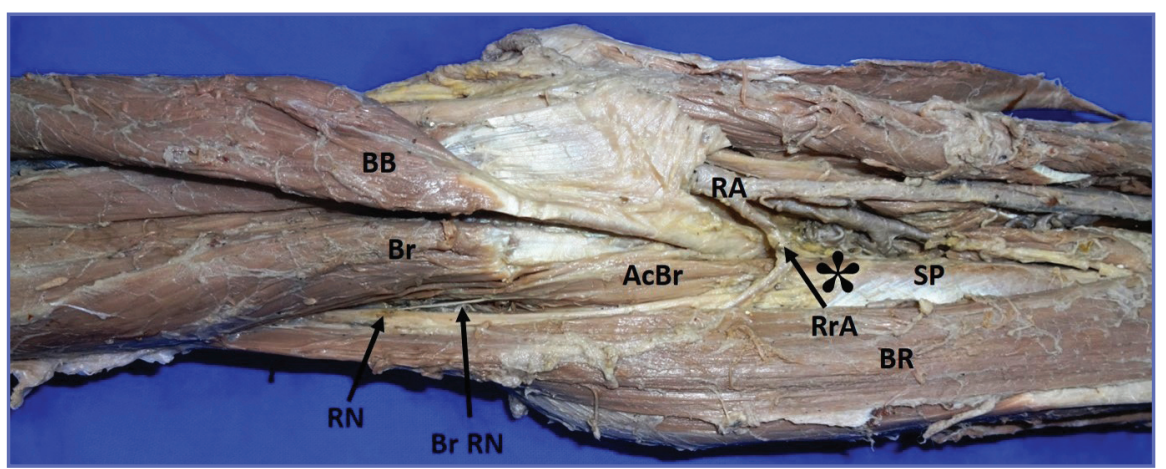

Figure 4. $\mathrm{AcBr}$ crossing deep to the RrA and inserting into the SP (15.2\%; type 3a). BB: biceps brachii; Br: brachialis; AcBr: accessory brachialis; BR: brachioradialis; SP: supinator; RA: radial artery; $\mathrm{RrA}$ : radial recurrent artery; $\mathrm{RN}$ : radial nerve; $\mathrm{Br} \mathrm{RN}$ : branch of radial nerve supplying $\mathrm{AcBr}$. *Insertion of $\mathrm{AcBr}$ into SP. 


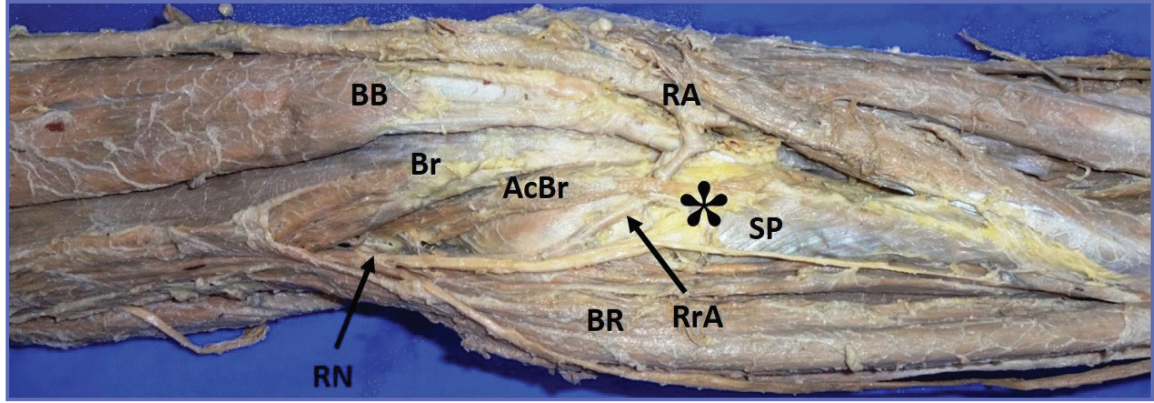

Figure 5. AcBr crossing superficial to the RrA and inserting into the SP (2.2\%; type 3b).

BB: biceps brachii; Br: brachialis; AcBr: accessory brachialis; BR: brachioradialis; SP: supinator; RA: radial artery; RrA: radial recurrent artery; RN: radial nerve. "Insertion of AcBr into SP.

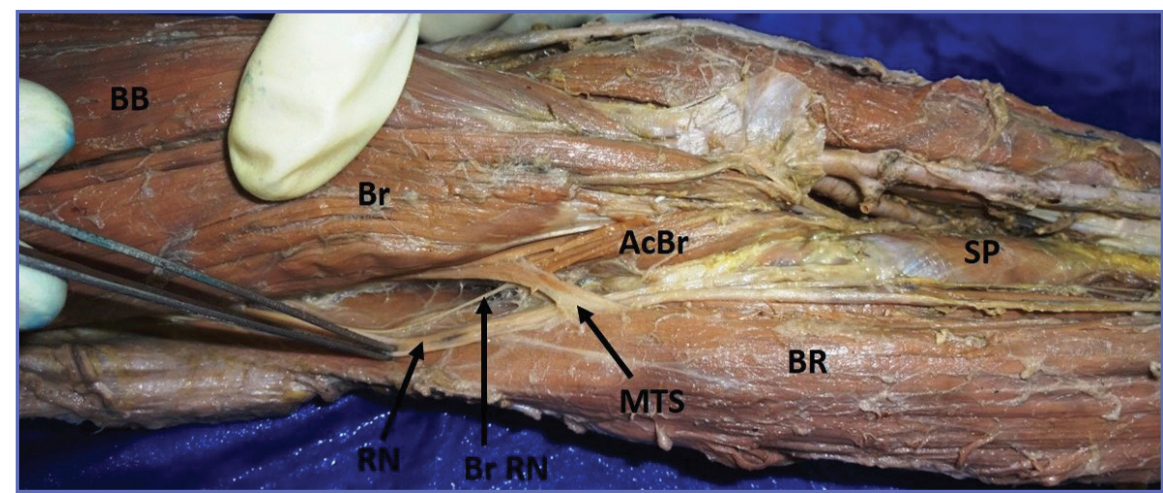

Figure 6. MTS from AcBr crossing over the RN to join the BR (2.2\%; type 4).

BB: biceps brachii; Br: brachialis; AcBr: accessory brachialis; BR: brachioradialis; SP: supinator; RN: radial nerve; $\mathrm{Br} \mathrm{RN}$ : branch of radial nerve supplying AcBr. MTS: musculotendinous slip.

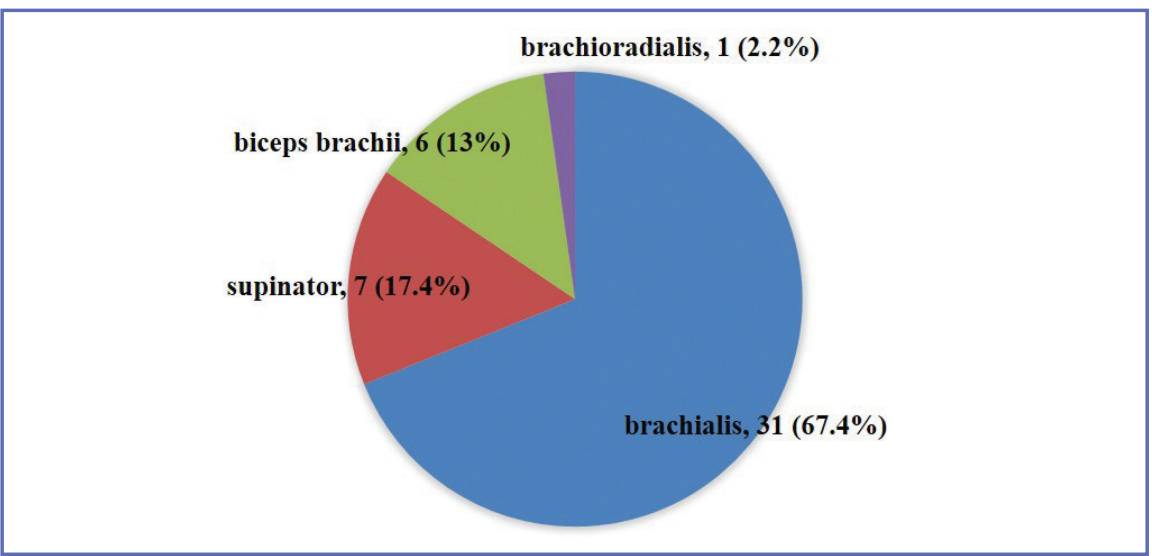

Figure 7. Frequency distribution of variability of insertion of $\mathrm{AcBr}$ observed in this study $(n=46)$. bud (13). These growth factors will help in the migration of myoblasts into the developing limb buds. The adhesion molecules which are expressed by the myoblasts are involved in the distribution of growth factors in a proper arrangement in the limb (14). The muscle primordia later get split into muscles of flexor and extensor compartments. This division is due to signaling by the connective tissue, which is derived by the lateral plate mesoderm. Postero-anterior development is under the control of WNT7A and the antero-posterior development is due to signalling of sonic hedgehog protein, which is secreted at the zone of polarizing activity in the posterior limb bud (12). Alteration in these pathways can lead to various anatomical variations in the muscular system. The muscle precursors which are found as different layers in the arm fuse to form a single muscle at a particular stage (13, 15). However, some primordial muscle cells go for apoptosis in spite of their contents as myofilaments (13). The failure of their apoptosis may contribute to the development of accessory muscles. It was reported that any changes in the structure of myotome or the somite, or in the distribution of adhesion molecules in the myoblasts may lead to muscular variations, which includes the accessory muscles (16). This is the developmental basis of the presence of accessory muscles.

In the present study, $\mathrm{AcBr}$ was observed in $54.8 \%$ of cases. AcBr muscle usually originates from the lateral intermuscular septum of arm and the fascia covering deltoid and triceps brachii muscles (17). Disparities in the morphology of brachialis though unusual, but are recorded in the literature that it may be divided into two or more parts, which blend with the brachioradialis, biceps brachii or pronator teres. The distal attachment of the $\mathrm{AcBr}$ into the shaft of radius below the bicipital tuberosity can hinder the flexion of the 
elbow joint at the ulnar component. This will further lead to difficulty in the pronation and supination of the forearm due to the variant $\mathrm{AcBr}$ muscle aponeurosis $(9,17)$. In the present study the $\mathrm{AcBr}$ has joined with the tendons of supinator, brachioradialis, brachialis and biceps brachii muscles. Here the $\mathrm{AcBr}$ may be enhancing the actions of the supinators of forearm. The AcBr reported by Pai et al. (6) inserted into the supinator and pronator teres after crossing the radial nerve thus contributing to the factors leading to radial tunnel syndrome. In the present study AcBr joined the supinator in 8 upper limbs $(17.4 \%)$, this insertion pattern may lead to narrowing of the radial tunnel during the supination of forearm thus leading to radial tunnel syndrome. In one of the specimen in the present study, the tendon of $\mathrm{AcBr}$ traversed superficial to the radial recurrent artery before inserting into the supinator $(2.2 \%)$, thus compressing the vessels in certain positions of the forearm. This type $3 \mathrm{~b}$ variety, may not cause ischemia due to the very good anastomosis around the elbow joint. In one of the specimens of the present study, musculotendinous fibres arising from the $\mathrm{AcBr}$ bridged across the radial nerve and merged with brachioradialis (type 4, 2.2\%). This bridge also narrows the radial tunnel and may contribute to the radial tunnel syndrome by compressing the radial nerve. In our previous study (18) with different anatomical specimens which was performed few years ago, it was observed that brachialis was giving muscular slips to the brachioradialis muscle. This was observed in $28.5 \%$ cases of the upper extremities. In that study (18) we classified the connecting bridges into split type, slip type and tendinous types. In all those cases, the intermuscular connection was oblique and entrapping the radial nerve. There have been instances of $\mathrm{AcBr}$ crossing the brachial artery and the median nerve $(7,8)$. This shows that the presence of AcBr could cause medial nerve entrapment in the forearm and compression of the brachial artery. We did not find any such compression pathology in our study. Nelluri et al. (17), has reported a case of $\mathrm{AcBr}$, which was crossing the radial artery, thus having the potential to compress it. The brachialis is usually supplied by the musculocutaneous nerve and radial nerve. The AcBr in the present study were all supplied by twigs from the radial nerve. It was described that, brachialis has dual heads of origin, superficial and deep. Between these two an internervous plane can be found, which can be utilized for the anterolateral surgical approach to the cubital articulation (9).

Akhtar et al. (16) reported the insertion of $\mathrm{AcBr}$ into the pronator teres muscle. But this morphological variant was not observed in the present study. George and Nayak (19) encountered a variation in which few fibres of brachialis merged with superficial muscles of front of forearm and to the olecranon process. Loukas et al. (7) observed an $\mathrm{AcBr}$ muscle, which was splitting to envelop the median nerve and finally inserted back to the brachialis tendon. Dharap (20) reported an accessory muscle, which was forming a tunnel for median nerve and brachial artery in the arm. Paraskevas et al. (4) and Vadgaonkar et al. (21) observed an AcBr muscle, which was merging with the medial intermuscular septum. These type of morphological variants are not observed in the present study. The AcBr inserting into the biceps brachii is termed as brachiobicipitalis (14). This was observed in 6 cases $(13 \%)$ in our specimens. This morphological variant can offer greater supination of the forearm and hand.

Mistry et al. (22) observed AcBr in only 5\% of their specimens. These muscles were inserting into the brachioradialis and bicipital aponeurosis. Khandey et al. (9) observed $\mathrm{AcBr}$ in only $3.5 \%$ of their cases. The present study is not agreeing with the incidence rate of $\mathrm{AcBr}$ with respect to these studies. We observed higher incidence of $\mathrm{AcBr}$ (54.8\%), which is not as per the descriptions of classical anatomical text books. The morphological knowledge about $\mathrm{AcBr}$ is important to understand the conditions like median nerve entrapment. The knowledge is also important during the surgical procedures around the elbow joint and brachial artery catheterization. This will also prevent misinterpretations during the radio diagnostic procedures and angiographic studies around the cubital fossa. It was reported that the tendon of $\mathrm{AcBr}$ muscle can be harvested for the reconstruction of annular ligament of the superior radioulnar joint, tibial collateral ligament of the knee joint and also in tendon transfer surgeries (6). The ruptured tendon of biceps brachii can be reconstructed by using the $\mathrm{AcBr}$, by transferring it to the radial tuberosity (22). The fibres of $\mathrm{AcBr}$ are also utilized in reconstruction of ulnar collateral ligament of the elbow joint. Since the muscles of forearm are not always available for the tendon transfer, if $\mathrm{AcBr}$ is present, this can be used as an alternative donor for the reconstruction of the flexor digitorum profundus and flexor pollicis longus muscles during the brachial plexus injury (23).

$\mathrm{AcBr}$ can alter the arthrokinematics of the shoulder joint, leading to pain during the mobility of the rotator cuff. The clinical testing of the brachialis muscle can be misinterpreted due to the presence of AcBr. The accessory muscles in the arm should not be misinterpreted as tumours during the reporting of the MRI in this region (24). They can be misinterpreted as soft tissue tumours like fibroma and neuro$\mathrm{ma}$ (14). Presence of $\mathrm{AcBr}$ and the variations in its insertion is definitely an important factor contributing to nerve compression syndromes like radial tunnel syndrome. The accessory muscles at the arm can be considered as the reason, whenever there is an entrapment neuropathy along with the brachial artery or brachial vein compression. Surgeries in the cubital fossa, especially elbow joint surgeries may get 
complicated due to the presence of AcBr. The percutaneous brachial approach is often preferred during the carotid and vertebral angiography. The knowledge about $\mathrm{AcBr}$ can prevent the complication in this procedure. The ultrasound guided brachial artery puncture is suggested in these situations and it should be considered (25).

We believe that the present study has provided additional information about the AcBr muscle. However, the limitation of the present study is that, since it is a cadaveric study, the biomechanics of the elbow joint and signs and symptoms of the entrapment neuropathy could not be assessed in this anatomical investigation. Future implications of this study include studying the $\mathrm{AcBr}$ in vivo by using ultrasound, MRI and electromyogram methods. Elastography is a newly established North American based technique to assess the tissue stiffness (26). The AcBr can be studied in vivo by using elastography and the stiffness of the arm can be evaluated. Analan and Ozdemir (26) reported that,

\section{REFERENCES}

1. Gessini L, Jandolo B, Pietrangeli A. Entrapment neuropathies of the median nerve at and above the elbow. Surg Neurol 1983;19:112-6.

2. Nakatani T, Tanaka S, Mizukami S. Bilateral four-headed biceps brachii muscles: the median nerve and brachial artery passing through a tunnel formed by a muscle slip from the accessory head. Clin Anat 1998;11:209-12.

3. Sookur PA, Naraghi AM, Bleakney RR, Jalan R, Chan O, White LM. Accessory muscles: anatomy, symptoms, and radiologic evaluation. Radiographics 2008;28:481-99.

4. Paraskevas G, Natsis K, Ioannidis O, Papaziogas B, Kitsoulis $P$, Spanidou S. Accessory muscles in the lower part of the anterior compartment of the arm that may entrap neurovascular elements. Clin Anat 2008;21:246-51.

5. Frazer EA, Hobson M, McDonald SW. The distribution of the radial and musculocutaneous nerves in the brachialis muscle. Clin Anat 2007;20:785-9.

6. Pai MM, Nayak SR, Vadgaonkar R, et al. Accessory brachialis muscle: a case report. Morphologie 2008;92:47-9.

7. Loukas M, Louis RG Jr, South G, Alsheik E, Christopherson C. A case of an accessory brachialis muscle. Clin Anat 2006;19:550-3.

8. Sirasanagandla SR, Potu BK. Clinical and functional significance of accessory brachialis muscle: A unique anatomical variant. Int J Morphol 2013;31:1383-5.

9. Khandey S. Morphology of brachialis muscle: variations and clinical significance. Int J Anat Res 2014;2:184-6.

10. Vadgaonkar R, Rai R, Nayak SR, D'Costa S, Saralaya V, Dhanya. An anatomical and clinical insight on brachialis with emphasis on portal's muscle. Rom J Morphol Embryol 2010;51:551-3.

11. Padulo J, Oliva F, Frizziero A, Maffulli N. Padulo J, Oliva F, Frizziero A, Maffulli N. Muscles, Ligaments and Tendons neurological disorders are not the only etiological factors for the muscle stiffness, few more factors like anatomical variations, posture, gender and exercise also have a role.

\section{CONCLUSIONS}

The present study offers the information about the incidence of $\mathrm{AcBr}$, variability in its insertion and relation with the surrounding neurovascular structures. The preoperative knowledge about $\mathrm{AcBr}$ is important to the orthopedicians, anaesthesiologists and radiologists. This can avoid the misinterpretation and subsequent complications. The details are essential for the accurate diagnosis and management of the musculoskeletal disorders of the arm.

\section{CONFLICT OF INTERESTS}

The authors declare that they have no conflict of interests.

Journal - Basic principles and recommendations in clinical and field Science Research: 2018 update. Muscles Ligaments Tendons J 2018;8(3):305-7.

12. Guéro S. Developmental biology of the upper limb. Hand Surg Rehabil 2018;37:265-74.

13. Saddler TW. Muscular system. In: Langman's Medical Embyology. 11th ed. New Delhi: Wolter's Kluwer (India) Pvt Ltd, 2010;pp. 147-154.

14. Mehta V, Yadav Y, Arora J, Kumar H, Suri RK, Rath G. Clinico-embryological perspective of a rare accessory brachial muscle with possible musculocutaneous nerve compression. Morphologie 2009;93:27-9.

15. Arey LB. Developmental Anatomy. In: A Textbook and Laboratory Manual of Embryology. 6th ed. Phildelphia: WB Saunders company, 1960;pp. 434-5.

16. Akhtar MJ, Fatima N, Kumar S, Kumar B, Kumar V. An accessory brachialis muscle associated with abnormal arrangement of structures in the cubital fossa. Int J Res Med Sci 2015;3:3907-10.

17. Nelluri V, Swamy RS, Nayak BS, Kumar N, Patil J. Bulky accessory brachialis muscle with abnormal aponeurosis: a case report. Proc Singapore Healthc 2016;25:249-51.

18. Tonse M, Pai MM, Prabhu LV, Murlimanju BV, Vadgaonkar R, Rao YL. Intermuscular connections in anterior brachium: its implications in radial nerve entrapment neuropathy. Muscles Ligaments Tendons J 2019;9:579-83.

19. George BM, Nayak SB. Median nerve and brachial artery entrapment in the abnormal brachialis muscle-a case report. Neuroanatomy 2008;7:41-2.

20. Dharap AS. An anomalous muscle in the distal half of the arm. Surg Radiol Anat 1994;16:97-9. 
21. Vadgaonkar R, Rai R, Ranade AV, Nayak SR, Pai MM, Lakshmi R. A case report on accessory brachialis muscle. Rom J Morphol Embryol 2008;49:581-3.

22. Mistry PN, Rajguru J, Dave MR. An anatomical insight into the morphology of the brachialis muscle and its clinical implications. Int $\mathrm{J}$ Anatom Radiol Surg 2021;10:AO16-AO20.

23. Bertelli JA, Ghizoni MF. Brachialis muscle transfer to reconstruct finger flexion or wrist extension in brachial plexus palsy. J Hand Surg Am 2006;31:190-6.
24. Leonello DT, Galley IJ, Bain GI, Carter CD. Brachialis muscle anatomy. A study in cadavers. J Bone Joint Surg Am 2007;89:1293-7.

25. Chuang YM, Luo CB, Chou YH, Cheng YC, Chang CY, Chiou HJ. Sonographic diagnosis and treatment of a median nerve epineural hematoma caused by brachial artery catheterization. J Ultrasound Med 2002;21:705-8.

26. Analan PD, Ozdemir H. Assessment of post-stroke biceps brachialis muscle stiffness by shear-wave elastography: a pilot study. Muscles Ligaments Tendons J 2020;10:531-5. 